\title{
On Starlike Functions Using the Generalized Salagean Differential Operator
}

\author{
Saliu Afis' ${ }^{1}$, Mashood Sidiq \\ ${ }^{1}$ Department of Mathematics, Gombe State University, Gombe, Nigeria \\ ${ }^{2}$ Department of Mathematics, University of Ilorin, Ilorin, Nigeria \\ Email: afis.saliu66@gmail.com,mashoodsidiq@yahoo.com
}

How to cite this paper: Afis, S. and Sidiq, M. (2016) On Starlike Functions Using the Generalized Salagean Differential Operator. Open Access Library Journal, 3: e2895. http://dx.doi.org/10.4236/oalib.1102895

Received: August 19, 2016

Accepted: September 11, 2016

Published: September 14, 2016

Copyright $\odot 2016$ by authors and Open Access Library Inc.

This work is licensed under the Creative Commons Attribution International License (CC BY 4.0).

http://creativecommons.org/licenses/by/4.0/

\section{Abstract}

In this paper we investigate the new subclass of starlike functions in the unit disk $U=\{z \in \mathbb{C}:|z|<1\}$ via the generalized salagean differential operator. Basic properties of this new subclass are also discussed.

\section{Subject Areas}

Mathematical Analysis

\section{Keywords}

Salagean Differential Operator, Starlike Functions, Unit Disk, Univalent Functions, Analytic Functions and Subordination

\section{Introduction}

Let $\mathbb{A}$ denote the class of functions:

$$
f(z)=z+a_{2} z^{2}+\cdots
$$

which are analytic in the unit disk $U=\{z \in \mathbb{C}:|z|<1\}$. Denote by

$$
S^{*}=\left\{f \in \mathbb{A}: R e \frac{z f^{\prime}(z)}{f(z)}>0, z \in U\right\} \text { the class of normalized univalent functions in } U \text {. }
$$

Let $g(z)=z+b_{2} z^{2}+\cdots \in \mathbb{A}$. We say that $f(z)$ is subordinate to $g(z)$ (written as $f \prec g$ ) if there is a function $w$ analytic in $U$, with $w(0)=0,|w(z)|<1$, for all $z \in U$. If $g$ is univalent, then $f \prec g$ if and only if $f(0)=g(0)$ and $f(U) \subseteq g(U)$ [1].

Definition 1 ([2]). Let $f \in \mathbb{A}, \lambda \in(0,1]$ and $n \in \mathbb{N}$. The operator $D_{\lambda}^{n}$ is defined by 


$$
\begin{aligned}
& D_{\lambda}^{0} f(z)=f(z), \\
& D_{\lambda}^{1}=(1-\lambda) f(z)+\lambda z f^{\prime}(z)=D_{\lambda} f(z), \\
& \quad \vdots \\
& D_{\lambda}^{n+1} f(z)=(1-\lambda) D_{\lambda}^{n} f(z)+\lambda z\left(D_{\lambda}^{n} f(z)\right)^{\prime}=D_{\lambda}\left(D_{\lambda}^{n} f(z)\right), z \in U .
\end{aligned}
$$

Remark 1. If $f \in \mathbb{A}$ and $f(z)=z+\sum_{k=2}^{\infty} a_{k} z^{k}$, then $D_{\lambda}^{n} f(z)=z+\sum_{k=2}^{\infty}[1+(k-1) \lambda]^{n} a_{k} z^{k}, z \in U$.

Remark 2. For $\lambda=1$ in (2), we obtain the Salagean differential operator.

From (2), the following relations holds:

$$
\left(D_{\lambda}^{n+1} f(z)\right)^{\prime}=\left(D_{\lambda}^{n} f(z)\right)^{\prime}+z \lambda\left(D_{\lambda}^{n} f(z)\right)^{\prime \prime}
$$

and from which, we get

$$
\frac{D_{\lambda}^{n+1} f(z)}{D_{\lambda}^{n} f(z)}=(1-\lambda)+z \lambda \frac{\left(D_{\lambda}^{n} f(z)\right)^{\prime}}{D_{\lambda}^{n} f(z)}
$$

Definition 2 ([3]). Let $f \in \mathbb{A}$, and $n \in \mathbb{N}_{0}$. Then

$$
\begin{aligned}
I_{\lambda}^{n} f(z) & =I_{\lambda}\left(I_{\lambda}^{n-1} f(z)\right)=\frac{1}{\lambda} z^{1-\frac{1}{\lambda}} \int_{0}^{z} t^{1-\frac{1}{\lambda}} I_{\lambda}^{n-1} f(t) \mathrm{d} t \\
& =z+\sum_{k=2}^{\infty} \frac{1}{[1+(k-1) \lambda]^{n}} a_{k} z^{k},
\end{aligned}
$$

with $I_{\lambda}^{0} f(z)=f(z)$.

This operator is a particular case of the operator defined in [3] and it is easy to see that for any $f \in \mathbb{A}, I_{\lambda}^{n}\left(D_{\lambda}^{n} f(z)\right)=D_{\lambda}^{n}\left(I_{\lambda}^{n} f(z)\right)=f(z)$.

Next, we define the new subclasses of $S^{*}$.

Definition 3. A function $f \in \mathbb{A}$ belongs to the class $S_{\lambda}^{n}$ if and only if

$$
\operatorname{Re} \frac{D_{\lambda}^{n+1} f(z)}{D_{\lambda}^{n} f(z)}>(1-\lambda), \quad \lambda \in(0,1]
$$

Remark 3. $S_{\lambda}^{0} \equiv S^{*}$.

Remark 4. $f \in S_{\lambda}^{n}$ if and only if $D_{\lambda}^{n} f(z) \in S^{*}$.

Definition 4. Let $u=u_{1}+u_{2} i, \quad v=v_{1}+v_{2} i$ and $\Psi$, the set of functions $\psi(u, v): \mathbb{C} \times \mathbb{C} \rightarrow \mathbb{C}$ satisfying.

i) $\psi(u, v)$ is continuous in a domain $\Omega$ of $\mathbb{C} \times \mathbb{C}$,

ii) $(1,0) \in \Omega$ and $\operatorname{Re} \psi(1,0)>0$,

iii) $\operatorname{Re} \psi\left(u_{2} i, v_{1}\right) \leq 0$ when $\left(u_{2} i, v_{1}\right) \in \Omega$ and $v_{1} \leq-\frac{1}{2}\left(1+u_{2}^{2}\right)$ for $z \in U$.

Several examples of members of the set $\Psi$ have been mentioned in [4] [5] and ([6], p. 27).

\section{Preliminary Lemmas}

Let $P$ denote the class of functions $p(z)=1+c_{1} z+c_{2} z^{2}+\cdots$ which are analytic in $U$ and satisfy $\operatorname{Re} p(z)>0, z \in U$. 
Lemma 1 ([5] [7]) Let $\psi \in \Psi$ with corresponding domain $\Omega$. If $P(\Psi)$ is defined as the set of functions $p(z)$ given as $p(z)=1+c_{1} z+c_{2} z^{2}+\cdots \quad$ which are regular in $U$ and satisfy.

i) $\left(p(z), z p^{\prime}(z)\right) \in \Omega$

ii) $\operatorname{Re} \psi\left(p(z), z p^{\prime}(z)\right)>0$ when $z \in U$.Then $\operatorname{Rep}(z)>0$ in $U$.

More general concepts were discussed in [4]-[6].

Lemma 2 ([8]). Let $\eta$ and $\mu$ be complex constants and $h(z)$ a convex univalent function in $U$ satisfying $h(0)=1$, and $\operatorname{Re}(\eta h(z)+\mu)$. Suppose $p \in P$ satisfies the differential subordination:

$$
p(z)+\frac{z p^{\prime}(z)}{\eta p(z)+\mu} \prec h(z), \quad z \in U .
$$

If the differential subordination:

$$
q(z)+\frac{z q^{\prime}(z)}{\eta q(z)+\mu}=h(z), \quad q(0)=1 .
$$

has univalent solution $q(z)$ in $U$. Then $p(z) \prec q(z) \prec h(z)$ and $q(z)$ is the best dominant in (6).

The formal solution of (6) is given as

$$
q(z)=\frac{z F^{\prime}(z)}{F(z)}
$$

where

$$
F(z)^{\eta}=\frac{\eta+\mu}{z^{\mu}} \int_{0}^{z} t^{\mu-1} H(t)^{\mu} \mathrm{d} t
$$

and

$$
H(z)=z \exp \left(\int_{0}^{z} \frac{h(t)-1}{t} \mathrm{~d} t\right)
$$

see [9] [10].

Lemma 3 ([9]). Let $\eta \neq 0$ and $\mu$ be complex constants and $h(z)$ regular in $U$ with $h^{\prime}(0) \neq 0$, then the solution $q(z)$ of $(7)$ given by (8) is univalent in $U$ if (i) $R e$ $\{G(z)=\eta h(z)+\mu\}>0$, (ii) $Q(z)=z \frac{G^{\prime}(z)}{G(z)} \in S^{*}$ (iii) $R(z)=\frac{Q(z)}{G(z)} \in S^{*}$.

\section{Main Results}

Theorem 1. Let $\lambda \in(0,1]$ and $h(z)$ a convex univalent function in $U$ satisfying $h(0)=1$, and $\operatorname{Re}\left(\frac{1-\lambda}{\lambda}+h(z)\right), \quad z \in U$. Let $f \in \mathbb{A}$. If $\frac{D_{\lambda}^{n+2} f(z)}{D_{\lambda}^{n+1} f(z)} \prec h(z)$, then $\frac{D_{\lambda}^{n+1} f(z)}{D_{\lambda}^{n} f(z)} \prec h(z)$

Proof. From (4), we have 


$$
\frac{D_{\lambda}^{n+2} f(z)}{D_{\lambda}^{n+1} f(z)}=(1-\lambda)+z \lambda \frac{\left(D_{\lambda}^{n+1} f(z)\right)^{\prime}}{D_{\lambda}^{n+1} f(z)} .
$$

If we suppose $\frac{D_{\lambda}^{n+2} f(z)}{D_{\lambda}^{n+1} f(z)} \prec h(z)$, we need to show that $\frac{D_{\lambda}^{n+1} f(z)}{D_{\lambda}^{n} f(z)} \prec h(z)$. Using the above equation and (4) and Remark 4, it suffices to show that if $\frac{z\left(D_{\lambda}^{n+1} f(z)\right)^{\prime}}{D_{\lambda}^{n+1} f(z)} \prec h(z)$, then $\frac{z\left(D_{\lambda}^{n} f(z)\right)^{\prime}}{D_{\lambda}^{n} f(z)} \prec h(z)$.

Now, let

$$
p=\frac{z\left(D_{\lambda}^{n} f(z)\right)^{\prime}}{D_{\lambda}^{n} f(z)} .
$$

Then

$$
z\left(D_{\lambda}^{n} f(z)\right)^{\prime \prime}+\left(D_{\lambda}^{n} f(z)\right)^{\prime}=p^{\prime}(z) D_{\lambda}^{n} f(z)+\left(D_{\lambda}^{n} f(z)\right)^{\prime} .
$$

By (2) and (3) we have

$$
\begin{aligned}
\frac{z\left(D_{\lambda}^{n+1} f(z)\right)^{\prime}}{D_{\lambda}^{n+1} f(z)} & =\frac{\lambda z p^{\prime}(z)+(1-\lambda) p(z)+\lambda p(z)^{2}}{(1-\lambda)+\lambda p(z)} \\
& =p(z)+\frac{z p^{\prime}(z)}{\frac{1-\lambda}{\lambda}+p(z)}
\end{aligned}
$$

Applying Lemma 2 with $\eta=1$ and $\mu=\frac{1-\lambda}{\lambda}$, the proof is complete. $\square$

Theorem 2. Let $\lambda \in(0,1 / 2]$ and $h(z)$ a convex univalent function in $U$ satisfying

$$
\begin{aligned}
h(0)=1, \text { and } \operatorname{Re}\left(\frac{1-\lambda}{\lambda}+h(z)\right)> & 0, z \in U \text {. Let } f \in \mathbb{A} \text {. If } f \in S_{\lambda}^{n} \text {, then } \\
& \frac{D_{\lambda}^{n+1} f(z)}{D_{\lambda}^{n} f(z)} \prec q(z)
\end{aligned}
$$

where

$$
q(z)=\frac{1+\sum_{k=0}^{\infty} \frac{(1+k)^{2}}{(1+\lambda k)} z^{k}}{1+\sum_{k=0}^{\infty} \frac{(1+k)}{(1+\lambda k)} z^{k}}
$$

is the best dominant.

Proof. Let $f \in S_{\lambda}^{n+1}$, then by Remark 4,

$$
\frac{z\left(D_{\lambda}^{n+1} f(z)\right)^{\prime}}{D_{\lambda}^{n+1} f(z)} \prec \frac{1+z}{1-z} .
$$

By (9), we have 


$$
p(z)+\frac{z p^{\prime}(z)}{\frac{1-\lambda}{\lambda}+p(z)} \prec \frac{1+z}{1-z},
$$

where

$$
p(z)=\frac{z\left(D_{\lambda}^{n} f(z)\right)^{\prime}}{D_{\lambda}^{n} f(z)} .
$$

To show that $\frac{z\left(D_{\lambda}^{n+1} f(z)\right)^{\prime}}{D_{\lambda}^{n} f(z)} \prec q(z)$, by Remark 4, it suffices to show that $p(z) \prec q(z)$.

Now, considering the differential equation

$$
q(z)+\frac{z q^{\prime}(z)}{\frac{1-\lambda}{\lambda}+q(z)}=\frac{1+z}{1-z}
$$

whose solution is obtained from (8). If we proof that $q(z)$ is univalent in $U$, our result follows trivially from Lemma 2 . Setting $\mu=\frac{1-\lambda}{\lambda}, \eta$ and $h(z)=\frac{1+z}{1-z}$ in Lemma 3, we have

i) $\operatorname{ReG}(z)=\operatorname{Re}(\mu+h(z))>0$,

ii) $Q(z)=\frac{z G^{\prime}(z)}{G(z)}=2 \lambda \frac{z}{(1+\beta z)(1-z)}$

where $\beta=2 \lambda-1$, so that by logarithmic differentiation, we have

$$
\frac{z Q^{\prime}(z)}{Q(z)}=\frac{1}{1-z}+\frac{1}{1+\beta z}-1
$$

Therefore, $R e \frac{z Q^{\prime}(z)}{Q(z)}>\frac{(1-2 \lambda)(1+\lambda)}{2 \lambda^{2}}>0$,

iii) $R(z)=\frac{Q(z)}{G(z)}=2 \lambda^{2} \frac{z}{(1+\beta z)^{2}}$

so that

$$
R e \frac{z R^{\prime}(z)}{R(z)}>\frac{1-\beta}{1+\beta}=\mu>0 .
$$

Hence, $q(z)$ is univalent in $U$ since it satisfies all the conditions of Lemma 3. This completes the proof. $\square$

Theorem 3. $S_{\lambda}^{n+1} \subset S_{\lambda}^{n}$.

Proof. Let $f \in S_{\lambda}^{n+1}$. By Remark 4

$$
R e \frac{z\left(D_{\lambda}^{n+1} f(z)\right)^{\prime}}{D_{\lambda}^{n+1} f(z)}>0 .
$$


From (9), let $\psi\left(p(z), z p^{\prime}(z)\right):=p(z)+\frac{z p^{\prime}(z)}{\frac{1-\lambda}{\lambda}+p(z)}$ with $p(z)=\frac{z\left(D_{\lambda}^{n} f(z)\right)^{\prime}}{D_{\lambda}^{n} f(z)}$ for $\Omega=\left[\mathbb{C}-\left\{-\frac{1-\lambda}{\lambda}\right\}\right] \times \mathbb{C}$. Conditions (i) and (ii) of Lemma 1 are clearly satisfied by $\psi$. Next, $\quad \psi\left(u_{2} i, v_{1}\right)=u_{2} i+\frac{v_{1}}{\frac{1-\lambda}{\lambda}+u_{2} i}$. Then $\quad \operatorname{Re} \psi\left(u_{2} i, v_{1}\right)=\frac{\frac{1-\lambda}{\lambda} v_{1}}{\left(\frac{1-\lambda}{\lambda}\right)^{2}+u_{2}^{2}} \leq 0 \quad$ if $v_{1} \leq-\frac{1}{2}\left(1+u_{2}^{2}\right)$. Hence, $\operatorname{Rep}(z)>0$. Using Remark 4, $\operatorname{Re} \frac{D_{\lambda}^{n+1} f(z)}{D_{\lambda}^{n} f(z)}>1-\lambda$ which complete the proof. $\square$

Corollary 1. All functions in $S_{\lambda}^{n}$ are starlike univalent in $U$.

Proof. The proof follows directly from Theorem 3 and Remark $4 . \square$

Corollary 2. The class $S_{1}^{n}$ "clone" the analytic representation of convex functions.

Proof. The proof is obvious from the above corollary and Definition 4. $\square$

The functions $f(z)=z+\frac{z^{2}}{2 !}+\frac{z^{3}}{3 !}+\cdots$ and $g(z)=z-\frac{z^{2}}{2 \times 2 !}+\frac{z^{3}}{3 \times 3 !}+\cdots$ are examples of functions in $S_{1}^{n}$.

Theorem 4. The class $S_{\lambda}^{n}$ is preserve under the Bernardi integral transformation:

$$
F(z)=\frac{c+1}{z^{c}} \int_{0}^{z} t^{c-1} f(t) \mathrm{d} t, \quad c>-1 .
$$

Proof. let $f \in S_{\lambda}^{n}$, then by Remark $4 D_{\lambda}^{n} f(z) \in S^{*}$. From (10) we get

$$
(c+1) f(z)=c F(z)+z F^{\prime}(z) \text {. }
$$

Applying $D_{\lambda}^{n}$ on (10) and noting from Remark 1 that $D_{\lambda}^{n}\left(z F^{\prime}(z)\right)=z\left(D_{\lambda}^{n} F(z)\right)^{\prime}$, we have

$$
\frac{z\left(D_{\lambda}^{n} f(z)\right)^{\prime}}{D_{\lambda}^{n} f(z)}=\frac{(c+1) z\left(D_{\lambda}^{n} F(z)\right)^{\prime}+z^{2}\left(D_{\lambda}^{n} F(z)\right)^{\prime \prime}}{c D_{\lambda}^{n} F(z)+z\left(D_{\lambda}^{n} F(z)\right)^{\prime}}
$$

Let $p(z)=\frac{z\left(D_{\lambda}^{n} F(z)\right)^{\prime}}{D_{\lambda}^{n} F(z)}$ and noting that $\frac{z^{2}\left(D_{\lambda}^{n} F(z)\right)^{\prime \prime}}{D_{\lambda}^{n} f(z)}=z p^{\prime}(z)+p(z)^{2}-p(z)$, we get

$$
\frac{z\left(D_{\lambda}^{n} f(z)\right)^{\prime}}{D_{\lambda}^{n} f(z)}=p(z)+\frac{z p^{\prime}(z)}{c+p(z)}
$$

Let $\psi\left(p(z), z p^{\prime}(z)\right)=: p(z)+\frac{z p^{\prime}(z)}{c+p(z)}$ for $\Omega=[\mathbb{C}-\{-c\}] \times \mathbb{C}$. Then $\psi$ satisfies all the conditions of Lemma 1 and so $\operatorname{Re} \frac{z\left(D_{\lambda}^{n} f(z)\right)^{\prime}}{D_{\lambda}^{n} f(z)}>0 \Rightarrow \operatorname{Re} \frac{z\left(D_{\lambda}^{n} F(z)\right)^{\prime}}{D_{\lambda}^{n} F(z)}>0$. By 
Remark $4 \quad F \in S_{\lambda}^{n}$

Theorem 5. Let $f \in S_{\lambda}^{n}$. Then fhas integral representation:

$$
f(z)=I_{\lambda}^{n}\left\{z \exp \left(\int_{0}^{z} \frac{p(t)-1}{t} \mathrm{~d} t\right)\right\}
$$

for some $p \in P$.

Proof. Let $f \in S_{\lambda}^{n}$. Then by Remark $4, D_{\lambda}^{n} F(z) \in S^{*}$ and so for some $p \in P$

$$
\frac{z\left(D_{\lambda}^{n} f(z)\right)^{\prime}}{D_{\lambda}^{n} f(z)}=p(z) .
$$

But $\frac{\mathrm{d}}{\mathrm{d} z}\left(\log \frac{D_{\lambda}^{n} f(z)}{\mathrm{z}}\right)=\frac{p(z)-1}{z}$, so that

$$
D_{\lambda}^{n} f(z)=z \exp \left(\int_{0}^{z} \frac{p(t)-1}{t} \mathrm{~d} t\right)
$$

Applying the operator in Definition 2, we have the result.

With $p(z)=\frac{1+z}{1-z}$, we have the extremal function for this new subclass of $S^{*}$ which is

$$
f_{\lambda}^{n}(z)=z+\sum_{k=2}^{\infty} \frac{k}{[1+(k-1) \lambda]^{n}} z^{k} .
$$

Theorem 6. Let $f \in S_{\lambda}^{n}$. Then

$$
\left|a_{k}\right| \leq \frac{k}{(1+(k-1) \lambda)^{n}}, \quad k \geq 2 .
$$

The function $f_{\lambda}^{n}(z)$ given by (13) shows that the result is sharp.

Proof. Let $f \in S_{\lambda}^{n}$, then by Remark $4, D_{\lambda}^{n} f(z) \in S^{*}$. Since it is well known that for any $f \in S^{*},\left|a_{k}\right| \leq k, k \geq 2$, then from Remark 1 we get the result. $\square$

Theorem 7. Let $f \in S_{\lambda}^{n}$. Then

$$
r\left(1-\mathcal{R}_{\lambda}^{n}\right)<|f(z)|<r\left(1+\mathcal{R}_{\lambda}^{n}\right)
$$

and

$$
1-r R_{\lambda}^{n} \leq\left|f^{\prime}(z)\right| \leq 1+r R_{\lambda}^{n}
$$

where

$$
\mathcal{R}_{\lambda}^{n}=\sum_{k=2}^{\infty} \frac{k}{[1+(k-1) \lambda]^{n}} \quad \text { and } \quad R_{\lambda}^{n}=\sum_{k=2}^{\infty} \frac{k^{2}}{[1+(k-1) \lambda]^{n}} .
$$

Proof. Let $f \in \mathbb{A}$. Then by Theorem 6, we have

$$
|f(z)| \leq|z|+\sum_{k=2}^{\infty}\left|a_{k}\right|\left|z^{k}\right|=r\left(1+\sum_{k=2}^{\infty} \frac{k}{[1+(k-1) \lambda]^{n}}\right)
$$


and

$$
|f(z)| \geq|z|-\sum_{k=2}^{\infty}\left|a_{k}\right|\left|z^{k}\right|=r\left(1-\sum_{k=2}^{\infty} \frac{k}{[1+(k-1) \lambda]^{n}}\right)
$$

for $|z|=r<1$.

Also, upon differentiating $f \in \mathbb{A}$, we get

$$
\left|f^{\prime}(z)\right| \leq 1+\sum_{k=2}^{\infty} k\left|a_{k}\right|\left|z^{k-1}\right| \leq 1+r \sum_{k=2}^{\infty} \frac{k^{2}}{[1+(k-1) \lambda]^{n}}
$$

and

$$
\left|f^{\prime}(z)\right| \geq 1-\sum_{k=2}^{\infty} k\left|a_{k}\right|\left|z^{k-1}\right| \geq 1-r \sum_{k=2}^{\infty} \frac{k^{2}}{[1+(k-1) \lambda]^{n}}
$$

for $|z|=r<1$. This complete the proof.

\section{Acknowledgements}

The authors appreciates the immense role of Dr. K.O. Babalola (a senior lecturer at University of Ilorin, Ilorin, Nigeria) in their academic development.

\section{References}

[1] Duren, P.L. (1983) Univalent Functions. Springer Verlag, New York Inc.

[2] Al-Oboudi, F.M. (2004) On Univalent Functions Defined by a Generalized Salagean Operator. International Journal of Mathematics and Mathematical Sciences, 27, 1429-1436. http://dx.doi.org/10.1155/S0161171204108090

[3] Faisal, I. and Darus, M. (2011) Application of a New Family of Functions on the Space of Analytic Functions. Revista Notas de Matemtica, 7, 144-151.

[4] Babalola, K.O. and Opoola, T.O. (2006) Iterated Integral Transforms of Caratheodory Functions and Their Applications to Analytic and Univalent Functions. Tamkang Journal of Mathematics, 37, 355-366.

[5] Miller, S.S. and Mocanu, P.T. (1978) Second Order Differential Inequalities in the Complex Plane. Journal of Mathematical Analysis and Applications, 65, 289-305. http://dx.doi.org/10.1016/0022-247X(78)90181-6

[6] Miller, S.S. and Mocanu, P.T. (2000) Differential Subordination, Theory and Applications. Marcel Dekker, 2000.

[7] Babalola, K.O. and Opoola, T.O. (2008) On the Coefficients of Certain Analytic and Univalent Functions. In: Dragomir, S.S. and Sofo, A., Eds., Advances in Inequalities for Series, Nova Science Publishers, 5-17. http://www.novapublishers.com

[8] Eenigenburg, P., Miller, S.S., Mocanu, P.T. and Read, M.O. (1984) On Briot-Bouquet Differential Surbordination. Revue Roumaine de Mathématiques pures et Appliquées, 29, 567573.

[9] Miller, S.S. and Mocanu, P.T. (1983) Univalent Solution of Briot-Bouquet Differential Equations. Lecture Notes in Mathematics, 1013, Springer Berlin/Heidelberg, 292-310.

[10] Srivastava, H.M. and Lashin A.Y. (1936) Some Applications of the Briot-Bouquet Differential Surbordination. Journal of Inequalities in Pure and Applied Mathematics, 37, 374-408. 